\title{
Mechanical phase mapping of the Taza meteorite using correlated high-speed nanoindentation and EDX
}

\author{
Jeffrey M. Wheelera) (1) \\ Laboratory for Nanometallurgy, Department of Materials Science, ETH Zürich, Zürich CH-8093, Switzerland \\ a)e-mail: jeff.wheeler@mat.ethz.ch \\ Received: 1 June 2020; accepted: 14 July 2020; published online: 11 February 2021
}

Meteorites have one of the most unique and beautiful microstructures, the Widmanstätten structure. This consists of large, elongated bands which form an intricate octahedral lace of crystalline metal. This structure makes meteorites an ideal case to demonstrate the capabilities of mechanical phase mapping using high-speed nanoindentation. In this work, the mechanical properties and composition of the Taza meteorite were mapped using $\sim 100,000$ indentations to statistically determine the properties of the individual phases. Five microstructural phases were characterized in this meteorite: Kamacite, Plessite, Tetrataenite, Cloudy Zone, and Schreibersite. Mechanical phase identification was confirmed using EDX measurements, and the first direct, pointto-point correlation of EDX and large-scale indentation maps was achieved. Mechanical phase maps showed superior phase contrast to EDX in two phases. An indentation property map or a mechanical phase map using a 2D histogram was used to visualize and statistically characterize the phases and identify trends in their relationships.

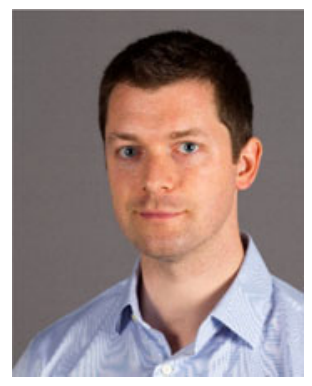

Jeffrey M. Wheeler is a leader in the areas of high temperature nanoindentation and micromechanical testing, particularly in situ in the SEM. He received his diploma in Materials Science and Engineering as a Camras/ NEXT scholar at the Illinois Institute of Technology in Chicago in 2005. He then joined the Department of Materials Science and Metallurgy at the University of Cambridge as an NSF Graduate Research Fellow where he earned his PhD on "Nanoindentation under Dynamic Conditions" in 2009. Subsequently, he joined the scientific staff at Empa, the Swiss Federal Laboratory for Materials Science and Technology, where he developed the first high temperature nanomechanical testing system capable of allowing direct visualization of testing in situ in the scanning electron microscope. He is currently a senior scientist within the Laboratory for Nanometallurgy in the Department of Materials Science at ETH Zürich in Switzerland, where he leads a research group on the Micromechanics of Materials. His research interests focus on the plasticity of strong materials at small length scales, the influence of ion irradiation of materials behavior, and large-scale indentation mapping.

\section{Introduction}

Meteorites are one of the most tangible aspects of outer space. With their irregular, ablated surfaces and intricate Widmanstätten microstructures, nickel-iron meteorites truly appear to be otherworldly. The internal microstructure and composition of meteorites have been intensively studied and classified by astro- and geo-chemists to study their origins and formation processes $[1,2]$. Nickel-iron meteorites can display a wide range of microstructures [3] depending on their nickel content, cooling rate, and tertiary composition [4]. These range from a pure body-centered cubic (BCC) iron phase (Kamacite) at low nickel concentrations to a pure facecentered cubic (FCC) phase (Taenite) at higher nickel concentrations. At the highest nickel concentrations, an ordered $\mathrm{L} 1_{0}$ intermetallic FeNi" phase called Tetrataenite can also form [4], which has interesting magnetic properties [5]. In between these concentrations, several different mixed phases occur forming either large Widmanstätten bands of Kamacite in a Taenite matrix or a spinodally-decomposed Plessite phase $[1,6]$. The width of these bands corresponds to extremely slow, radiative cooling over millions of years [4]. In addition 
to these majority phases, relatively large precipitates can also form by carbon, phosphorus, and sulfur segregation during cooling [4].

These exotic microstructures have long made meteorites attractive samples for demonstrating new characterization techniques, particularly analytical techniques [7]. However, other than microhardness testing in the 1950s-1970s [8, 9, 10], relatively few studies [11] have been performed to characterize the mechanical behavior of these unique pieces of other worlds. With the advent of modern, high speed nanoindentation techniques $[12,13,14]$, it is now possible to map the mechanical features of materials over square millimeters of area with micron-scale resolution in a reasonable amount of time. Using statistical analysis [15] or machine learning [16, 17], the properties of each of these phases can be extracted. This has been applied on a range of materials from cemented carbides [13, 18, 19], steels [20], and thermal barrier coatings [21] to actual cements $[22,23]$. By combining this technique with elevated temperature capable systems, the activation parameters of individual phases can also be extracted [24], or using a combinatorial approach on a diffusion couple, entire slices of phase diagrams can be interrogated at once [25]. High-speed nanoindentation mapping is still being actively developed, but it promises to become a ubiquitous tool for mechanical microscopy.

One area with significant potential for growth in highspeed nanoindentation mapping is correlative testing in combination with analytical techniques such as energy-dispersive $\mathrm{X}$-ray spectroscopy (EDX) and electron backscatter diffraction (EBSD). The combination of indentation mapping and EDX allows for several exciting applications. Chemical and mechanical degradation could be simultaneously quantified in multicomponent material structures in demanding environments, e.g., thermal barrier coatings after thermal cycling [21]. New understanding of the relationships between composition and mechanical behavior in materials with complex chemistries, such as cements, could be developed. Combinatorial studies [25] could be conducted in a more automatic fashion with the data from different phases or compositions effectively labeled by correlative analytic datasets, allowing easier phase identification and deconvolution. There is a broad scope for the application of these correlative techniques, but many challenges in data processing and analysis still remain.

In this work, high-speed nanoindentation is combined with EDX to map the mechanical properties of the different phases of a fragment of the Taza nickel-iron meteorite. To the author's knowledge, this is the first study which combines statistical, large-area nanoindentation and EDX with a point-to-point correlation. This meteorite has a rich microstructure consisting of at least five phases and provides an excellent case study for demonstrating this technique. Indentation mapping values are
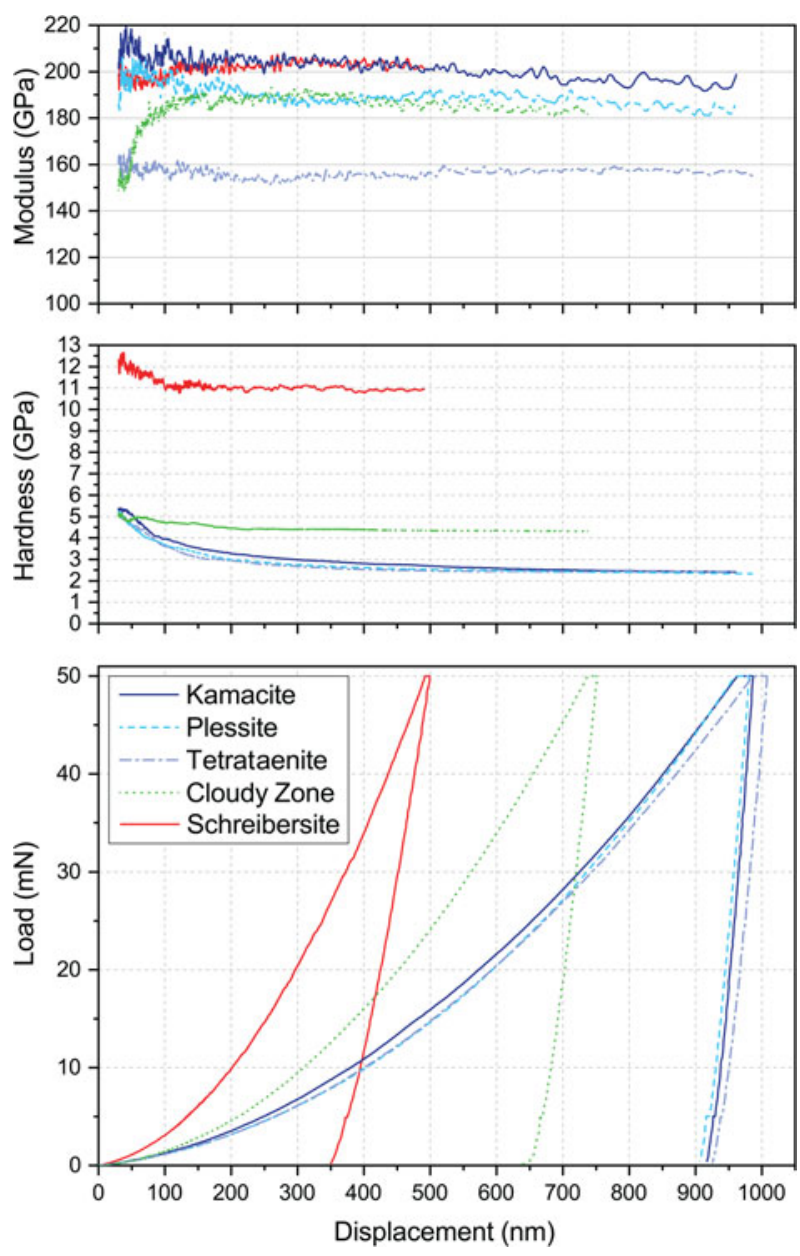

Figure 1: Representative curves for load, hardness, and modulus as a function of displacement from CSM indentations in each phase in the Taza meteorite.

compared to conventional depth-sensing indentation methods, and correlative EDX composition values are compared with literature and spot EDX measurements for each phase. Various statistical visualizations for the data are presented, and their benefits and limitations are discussed.

\section{Results}

\section{Individual indentations}

After preliminary indentation maps had identified the various phase regions in the sample, individual indentations using continuous stiffness measurement (CSM) were carefully placed in each phase region to characterize their modulus and hardness as a function of indentation depth. However, as this is the more conventional characterization method, I present these results first. Figure 1 shows representative load-displacement curves and hardness and modulus as a function of penetration depth for each phase in the Taza meteorite.

The Schreibersite, $(\mathrm{Fe}, \mathrm{Ni})_{3} \mathrm{P}$, precipitates are seen to be the hardest of all the phases, but interestingly Schreibersite has a 

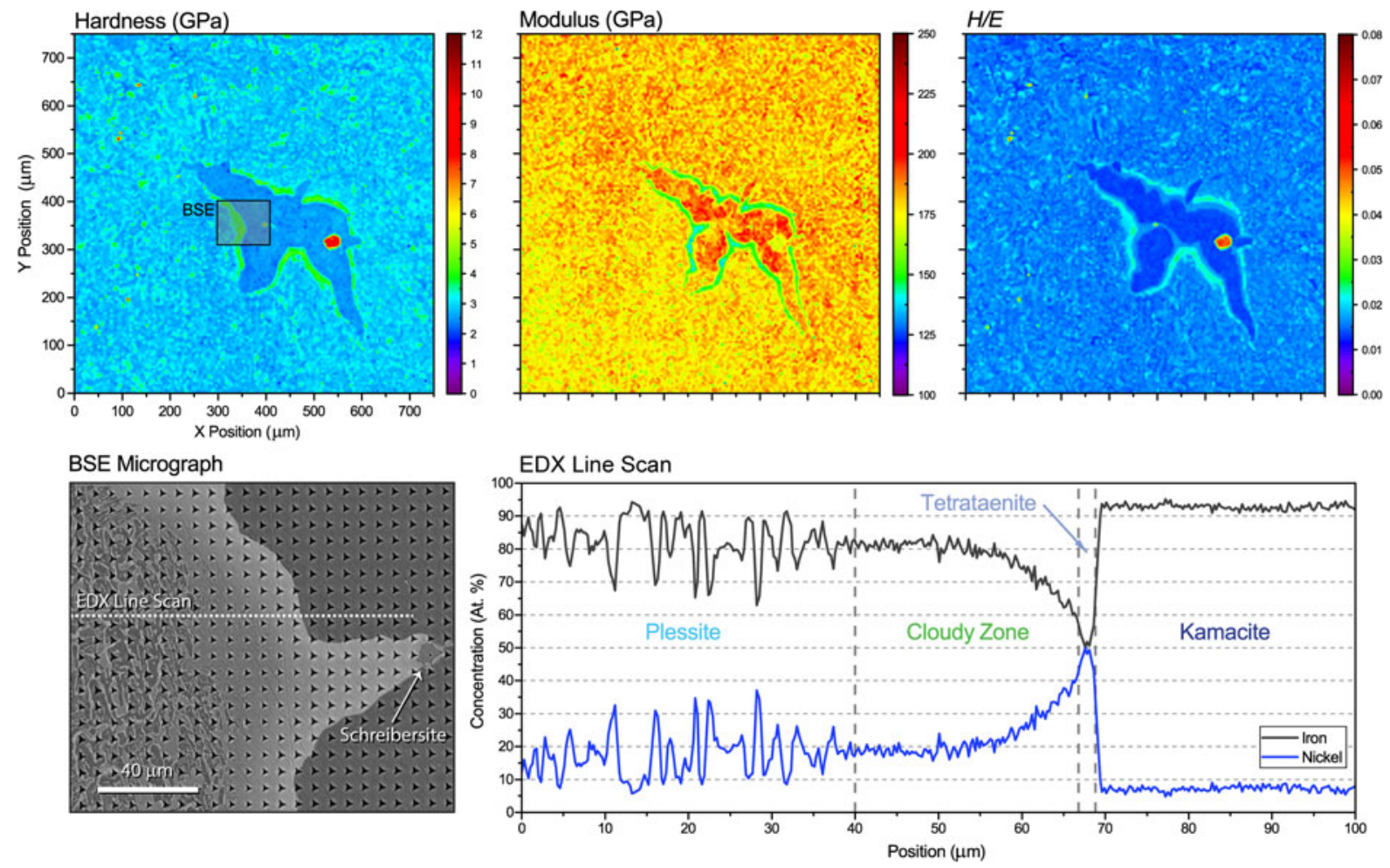

Figure 2: Comparison of indentation property maps of hardness, modulus, and H/E ratio with a local EDX line scan in a selected area investigated using BSE microscopy and indicated on the hardness map.

nearly identical modulus with the Kamacite phase that it precipitates inside. The Cloudy Zone phase is the next hardest phase with a hardness between 4 and $5 \mathrm{GPa}$, and it also has a nearly identical modulus trend with the Plessite phase. This is not too surprising, given the Cloudy Zone and Plessite phases are both composite microstructures made from the same two phases: Tetrataenite and Kamacite. The higher hardness and relatively small indentation size effect of the Cloudy Zone is attributed to the finer structure of the nanocomposite microstructure. Kamacite, Plessite, and Tetrataenite all possess nearly identical hardness trends, but the ordered $\mathrm{Ll}_{0}$ intermetallic Tetrataenite has a markedly lower modulus.

\section{EDX line scan correlation}

In Fig. 2, a small cluster of softer Kamacite bands can be seen in a harder, disordered Plessite matrix in the Taza meteorite, along with several hard Schreibersite precipitates. On closer examination, it can be seen that the soft bands in the hardness map contain an inner ring of lower modulus material and an outer halo of harder material. These consist of the inner Tetrataenite layer and the outer hard Cloudy Zone phase. These two layers appear quite distinct in the hardness-to-elastic modulus ratio, or $H / E$, map. These phases appear continuous in EDX scans, but discretely appear in mechanical phase maps. Good correlation is seen between the hardness and
H/E maps, but the hardness and modulus maps do not show the same 1:1 correlation between features, particularly in the Plessite matrix. This arises from the relatively similar moduli observed for the majority of phases, such that indents whose elastic zones overlap both phases either laterally or below the indentation measure a composite modulus which may not correspond to the surface phases. The plastic zones, which give rise to hardness values, are significantly more confined and give more local measurements corresponding to the surface phases.

The backscattered electron (BSE) micrograph shown in Fig. 2, which corresponds to the labeled shaded area in the hardness map, reveals the fine structure of the spinodally decomposed Plessite phase on the left side of the micrograph. Moving towards the right across the micrograph along the EDX line scan, the strong fluctuation in BSE contrast and elemental composition gives way to a smoother gradient corresponding to the Cloudy Zone phase [6]. This consists of a natural gradient nanocomposite material, whose modulation period becomes smaller than the EDX resolution. The hardness of this nanocomposite from the outer space increases as the nickel concentration increases, which relates to an increasingly fine nanocomposite structure with Tetrataenite in a fine Kamacite matrix. Moving further to the right, the ratio of iron and nickel reaches the 1:1 value necessary for the pure $\mathrm{FeNi}^{\prime \prime}$ Tetrataenite phase [6]. This corresponds to the drop in modulus seen in the modulus map. Finally, the pure Kamacite phase, consisting of 


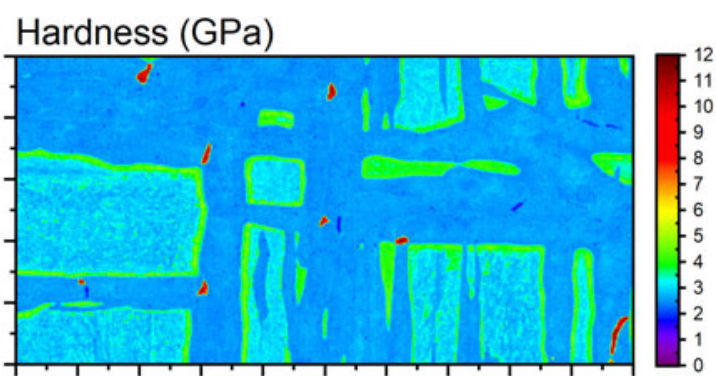

\section{Modulus (GPa)}
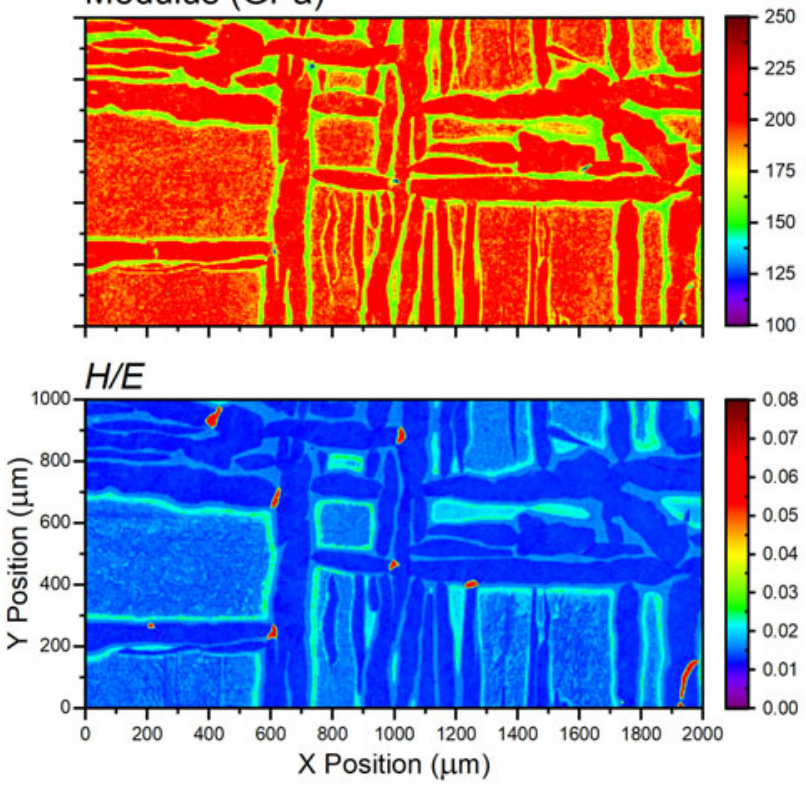

Iron (at. \%)
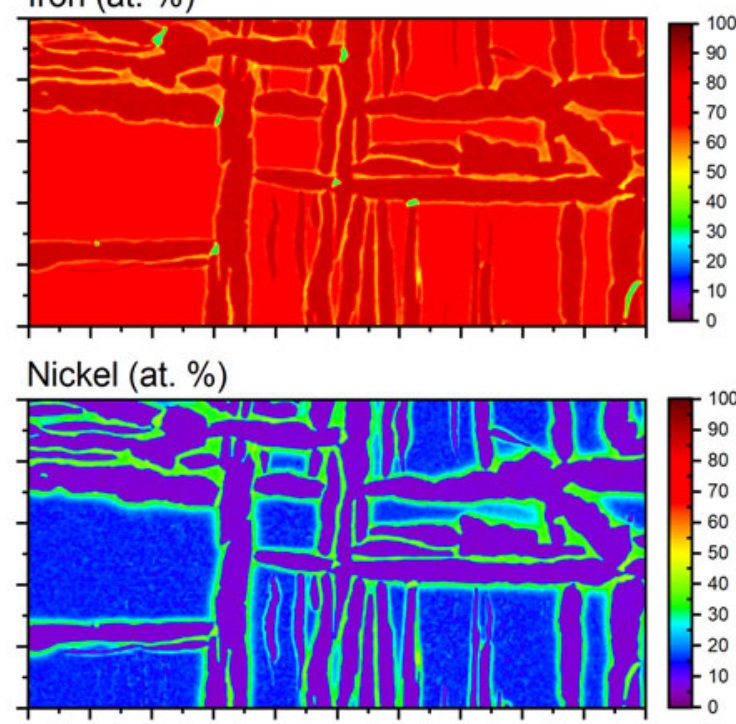

\section{Phosphorus (at. \%)}

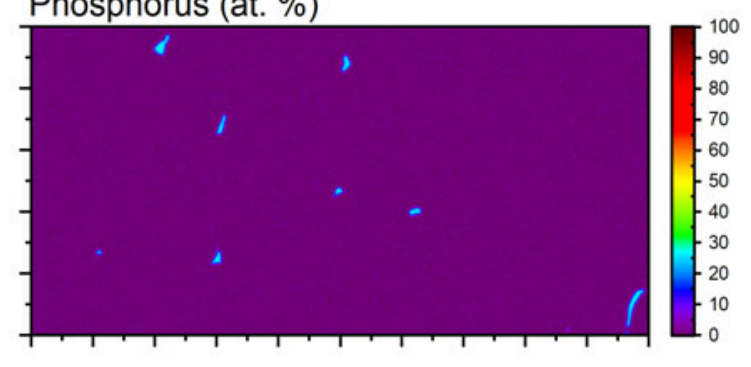

Figure 3: Large-area indentation property maps of a region of the Taza meteorite featuring numerous Kamacite bands with corresponding composition maps of iron, nickel, and phosphorus extracted from EDX data.

BCC iron, is observed and corresponds to the highest values observed in the modulus map.

Interestingly, a small precipitate of Schreibersite can also be observed in the BSE micrograph in Fig. 2. A single, smaller indent can be seen near the center of this $\sim 7 \mu \mathrm{m}$ diameter precipitate, which corresponds to the small peak in hardness in the shaded region in the hardness map. This single point might have been dismissed merely as noise, but this micrograph validates its veracity. This illustrates the accuracy of this mapping technique, despite its high speed.

\section{Large-area mechanical phase map}

The previous section described the basic features observed in the microstructure of the Taza meteorite in terms of the indentation results. This included a conventional correlation between mechanical indentation data and analytical EDX data. In this section, rather than correlating a few dozen indents to an EDX line scan, a full 1:1 correlation is achieved between indentation and EDX maps consisting of 80,000 points apiece (Fig. 3). This allows the first direct correlation between micron-scale mechanical and compositional property maps over square millimeters of area.

As it was not trivial to align and extract an arbitrarily/sized matrix of data using the EDX software, an oversampled EDX map was obtained instead, and the data were extracted using a custom piece of LabView software to align the data matrices and extract an average value of the EDX data within a set radius $(1.75 \mu \mathrm{m})$ of each indent's coordinates to determine corresponding 1:1 matrices for indentation and EDX data. For narrower phases, and shallower/narrower indentations in hard phases, this results in an averaging of EDX data over a larger area than the indentation's plastic zone, but the radius was chosen to correspond to the $\sim 3.5 \mu \mathrm{m}$ width of a $500 \mathrm{~nm}$ deep indentation performed with a Berkovich indenter. This size compares to the indents performed in the softest phase, Kamacite. If the indentation mapping techniques were able to be performed to a set target depth, rather than only a target maximum load, then higher resolution mapping of multiple phases would be possible with less averaged EDX data.

The features in the maps in Fig. 3 correspond well with the observations from Fig. 2 with Kamacite bands showing the 


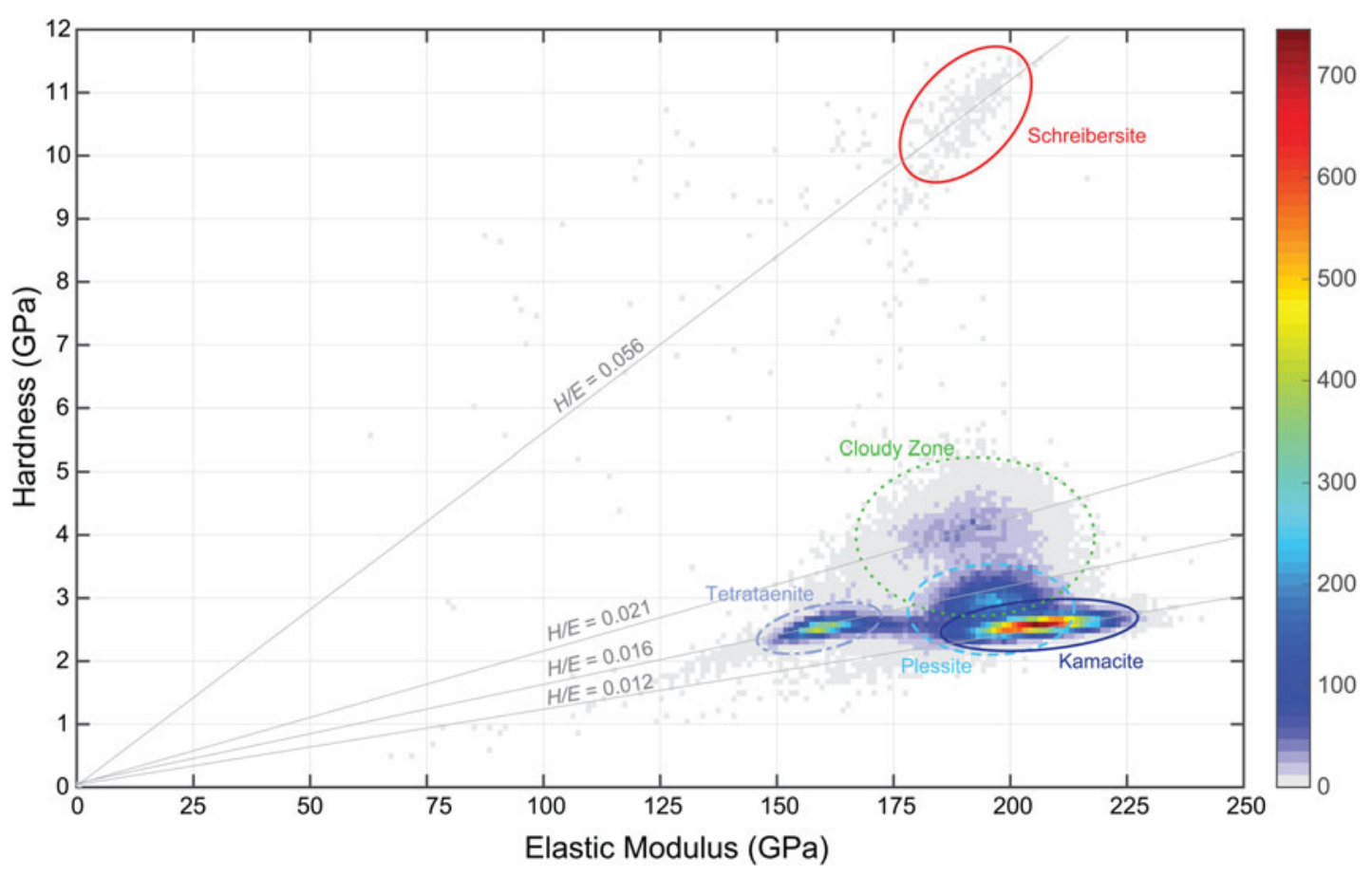

Figure 4: $2 D$ histogram of hardness versus elastic modulus from results shown in Fig. 3 with peaks corresponding to the phases labeled.

lowest hardness and highest modulus etc. However, the relatively richness of features in Fig. 3 is much greater due to its larger area and the increased density of Kamacite bands in that location. Furthermore, a much greater number of hard Schreibersite precipitates can be seen, which correspond to local hotpots in the phosphorus map. The correlative EDX maps allow a few additional trends to be observed. In general, decreased modulus corresponds to the high nickel concentrations of the Tetrataenite phase. Moreover, the true utility of the $H / E$ map is highlighted, as it is the only indentation map which allows easy observation of all five phases. In the $H / E$ map, both the Cloudy Zone and Tetrataenite phases can be clearly differentiated by their mechanical behavior. This is a notable feat, since the boundary between these phases is continuous in the EDX maps.

\section{Discussion}

\section{Statistical analysis}

Typical statistical analysis of large numbers of indentations [15] involves a blind deconvolution of a single indentation property: hardness or modulus. This involves fitting several Gaussian distributions to approximate a cumulative distribution function extracted from the property to be deconvoluted. This has been used with good success for a variety of materials where the distribution of the phases is somewhat random: cemented carbides [13, 18, 19], steels [20], and cements [22, 23]. Another technique is to use multivariate $K$-means cluster analysis [26], which incorporates several properties and performs an error minimization to split the data into several clusters which can be individually evaluated. This has been demonstrated on large nanoindentation datasets on thermal barrier coatings [21] and high entropy alloys [24].

In this work, I applied a simpler, more visual method for separating the different phases. By plotting the data from the 80,000 indentations shown in Fig. 3 in a 2D histogram of the hardness and elastic modulus, the phases can be easily visualized in property space (Fig. 4). This sort of plot has been used in previous work [25] to characterize the different phases in an $\mathrm{Al}-\mathrm{Cu}$ diffusion couple. In that work, the plot showed good correlation with values extracted using the analysis of Ulm et al. [15], demonstrating its utility for rapid visualization. All five phases can clearly be observed as clusters in this plot, and the regions of overlap between phases correspond to physical overlaps, where individual indentations occurred on the interfaces between phases. The strongest peak is clearly seen to be the Kamacite phase, whereas the Schreibersite values form more of a diffuse cloud than a strong peak. The main four phases all show significant overlap, due to their large number of interfaces from the intricate microstructure, shown in Fig. 3, resulting in a large number of indentations straddling two phases.

The 2D histogram plot is also revealing for why the hardness and modulus plots in Fig. 3 do not clearly show the variation between all of the phases, while the $H / E$ plot does allow all the phases to be visualized. If we examine the variation in properties purely from the modulus axis, the way we would 

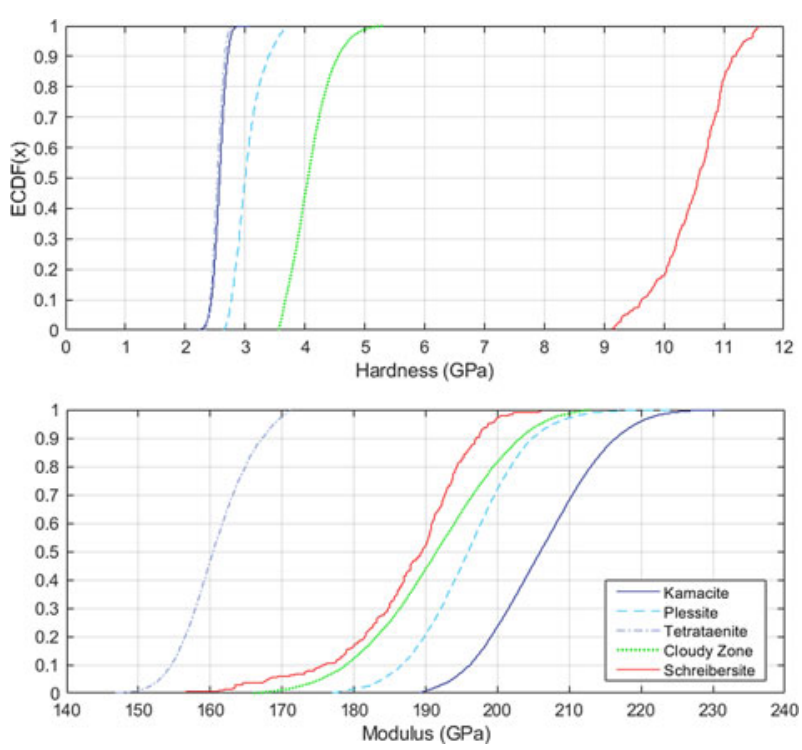

Figure 5: Empirical cumulative distribution functions for each phase in the Taza meteorite.

in a 1D histogram of modulus, then near complete overlap occurs for Schreibersite, Cloudy Zone, and Plessite. Only Tetrataenite and to a lesser extent Kamacite can be differentiated, as seen in Fig. 3. From the hardness axis, only the Cloudy Zone and Schreibersite phases remain distinct from the main cluster. However, despite the overlap in hardness and modulus, in this case, the $H / E$ ratios for each phase are quite distinct, as labeled in Fig. 3, resulting in the excellent phase contrast in that plot. This might not always be the case, as one can easily imagine a string of phases which all fall on the same $H / E$ ratio in the same way that these phases are aligned on the modulus axis. However, in every case, these phases would be distinct when plotted in a $2 \mathrm{D}$ histogram similar to Fig. 4, unless they were mechanically identical.

The 2D histogram also allows easy segregation of data for the more discrete phases: Schreibersite and Tetrataenite. Datapoints corresponding to those phases were easily separated, and their values evaluated to construct their empirical cumulative distribution functions shown in Fig. 5. However, the Cloudy Zone, Plessite, and Kamacite phases show significantly more overlap. To address these phases, the large numbers of indents over the large area shown in Fig. 3 were beneficial, since even a relatively small selected area could yield several thousand datapoints for analysis. Large areas of the Plessite phase were easily identified and selected by their positions. Similarly, large areas of Kamacite and Tetrataenite could also be selected, and then the Kamacite values isolated by removing the lower modulus values corresponding to the Tetrataenite phase. Thus, only the Cloudy Zone values needed further deconvolution. This was finally accomplished by using a hardness threshold of $3.6 \mathrm{GPa}$, which was the upper extent of the Plessite phase. This somewhat truncates the fit of the Cloudy zone phase, which noticeably overlaps with Plessite. However, the values in Table 1 extracted from both statistical and conventional indentations show good agreement.

While this manual/graphical method of separating the data for statistical analysis has the advantages of user supervision and confidence, it also carries the influence of human bias. The sum of the number of datapoints, $N$, investigated in each phase is $\sim 90 \%$ of the total indentations performed in this map. The unevaluated remainder consists of smaller regions of Plessite, which were not manually selected for analysis due to the availability of larger areas, intermediate indentations which fell between clusters and outliers. While this was sufficient in this case, ideally all data should be evaluated using a standard method. This highlights the need for further development to establish readily accessible, statistical/machine learning tools to facilitate the identification of phases. Some progress has been made in this area by applying multivariate $K$-means cluster analysis [26], but identifying appropriate standard methods for this remains a challenge for the nanoindentation mapping community. One avenue for improvement in phase identification is by increasing the parameter space to include correlative analytical techniques, e.g., EDX and/or EBSD, to add compositional and/or structural information to the mechanical data for each indentation.

\section{Correlation with EDX measurements}

As shown in the previous sections, visual correlations of the phase compositions and mechanical properties have been achieved with the EDX line (Fig. 2) and area (Fig. 3) scans.

TABLE 1: Average indentation values and standard deviations extracted from the conventional indentations in Fig. 1 and statistical results extracted from Fig. 5 .

\begin{tabular}{|c|c|c|c|c|c|c|c|c|c|}
\hline \multirow[b]{2}{*}{ Phase } & \multicolumn{4}{|c|}{ Conventional indentation } & \multicolumn{5}{|c|}{ Statistical indentation } \\
\hline & Hardness & S D & Elastic modulus & SD & Hardness & SD & Elastic modulus & SD & $N$ \\
\hline Kamacite & 2.555 & 0.198 & 199.5 & 6.852 & 2.572 & 0.106 & 206.2 & 7.778 & 35,629 \\
\hline Plessite & 2.879 & 0.231 & 185.8 & 3.774 & 3.043 & 0.233 & 195.9 & 7.215 & 18,972 \\
\hline Tetrataenite & 2.390 & 0.214 & 152.6 & 7.527 & 2.538 & 0.093 & 160.6 & 4.756 & 10,591 \\
\hline Cloudy Zone & 4.344 & 0.527 & 186.6 & 6.124 & 4.090 & 0.334 & 191.3 & 9.223 & 6856 \\
\hline Schreibersite & 11.03 & 0.674 & 190.8 & 14.46 & 10.49 & 0.576 & 187.4 & 9.010 & 249 \\
\hline
\end{tabular}




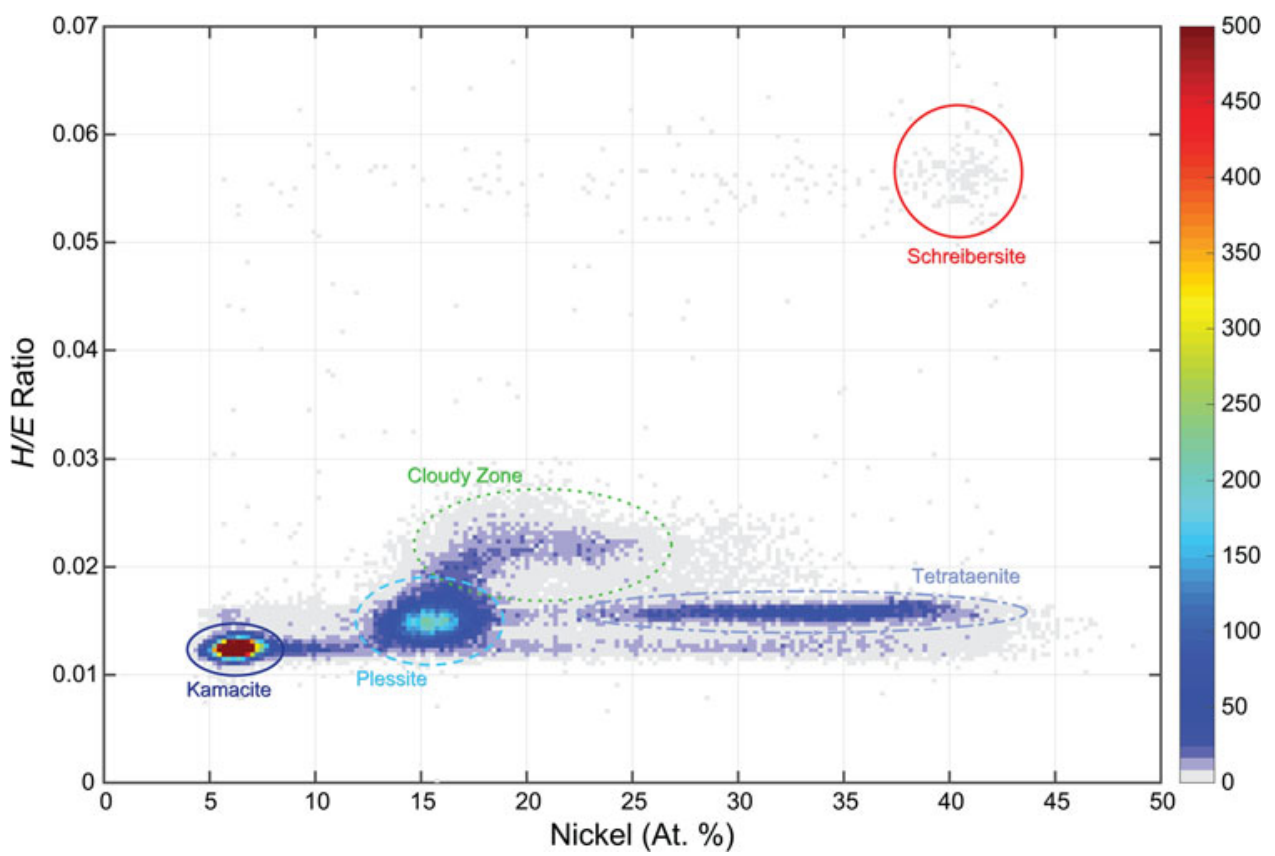

Figure 6: $2 \mathrm{D}$ histogram of $H / E$ ratio as a function of nickel content from the data in Fig. 3 with peaks corresponding to individual phases labeled.

The idea of correlating EDX and nanoindentation data is not novel, and it has been extensively applied previously to identify individual phases of interest in the microscope for manual phase identification or to qualitatively show the relationship between an EDX line scan and the hardness fluctuations in an indentation map [27]. For samples with smooth compositional gradients like diffusion couples/multiples [25, 28, 29, 30], quantitative correlation has also been achieved by fitting mathematical functions which describe compositional trends to a function of position to establish the relationship between indentation data and composition. Qualitative comparisons of larger-scale indentation maps with EDX [22] and EBSD [12] area scans of the same regions have also been achieved previously. However, the quantitative correlation of large-scale indentation data and EDX maps with 1:1 correlation for each datapoint in Cartesian space has not been achieved before. This truly allows quantitative mapping of mechanical properties as a function of composition, regardless of the feature or phase morphology or nature of the property gradients. This makes Fig. 4 unique, as it is likely the largest, point-for-point correlated map of its type to be achieved to date.

The direct correlation of nanoindentation and EDX data allows any nanoindentation parameter to be plotted as a function of composition, e.g., elastic modulus as a function of the Fe:Ni ratio or the hardness as a function of carbon content. However, as discussed above, the most descriptive indentation parameter for this particular dataset is the $H / E$ ratio, as it allows easy discrimination of all five phases present, and the most significant compositional influence on the phases in nickel-iron meteorites is the nickel content. Therefore, Fig. 6 was constructed to show the variation of the $H / E$ ratio with nickel content. At first glance, the choice of the $2 \mathrm{D}$ histogram again for this data appears strange, as it is more conventional to observe trends between hardness and composition represented as a line plot, as typically shown for solid solution strengthening investigations within single phases. However, in the case of

TABLE 2: Summary of compositional and structural parameters of the phases investigated [1, 4, 6, 31].

\begin{tabular}{|c|c|c|c|c|c|}
\hline Phase & Formula & Nickel content (at. \%) & Crystal system & Space group & Cell parameters $(\AA)$ \\
\hline Kamacite & $\mathrm{a}-(\mathrm{Fe}, \mathrm{Ni})$ & $0-7$ & $\mathrm{BCC}$ & $\operatorname{Im} 3 m$ & $a=2.86$ \\
\hline Plessite & $a-(\mathrm{Fe}, \mathrm{Ni})+\gamma-(\mathrm{Ni}, \mathrm{Fe})$ & $\sim 15$ & - & - & - \\
\hline Taenite & $\gamma-(\mathrm{Ni}, \mathrm{Fe})$ & $25-40$ & FCC & $\mathrm{Fm} 3 \mathrm{~m}$ & $a=7.146$ \\
\hline Tetrataenite & $\mathrm{FeNi}^{\prime \prime}$ & $\sim 49-52$ & $\mathrm{~L} 1_{0}$ Tetragonal & $\mathrm{P} 4 / \mathrm{mmm}$ & $\begin{array}{l}a=2.533 \\
c=3.582\end{array}$ \\
\hline Cloudy Zone & $a-(\mathrm{Fe}, \mathrm{Ni})+\mathrm{FeNi}^{\prime \prime}$ & $\sim 20-45$ & - & - & - \\
\hline Schreibersite & $(\mathrm{Fe}, \mathrm{Ni})_{3} \mathrm{P}$ & $\sim 18-42$ & Tetragonal & $\overline{4}$ & $\begin{array}{l}a=9.052 \\
c=4.47\end{array}$ \\
\hline
\end{tabular}


this multiphase meteorite, compositional gradients are quite local between the phase boundaries, so the trends in mechanical properties versus composition appear as more discrete clusters rather than trendlines. Figure 6 shows strong peaks/ clusters for all five phases, as labeled.

On comparing the literature values for the nickel content of the different phases (Table 2) with the values seen in Fig. 6, several interesting trends emerge. The peak for Kamacite is the strongest and occurs between 6 and 7 at.\% nickel, in good agreement with the EDX line scan in Fig. 2. Plessite shows the next strongest peak at $\sim 15$ at.\% nickel, again within the expected compositional range from the EDX line scan, and as the majority phase of the meteorite, it is also consistent with the overall nickel concentration for the meteorite (Table 3). Schreibersite shows a nickel concentration of $\sim 40 \%$ in good agreement with the point EDX quantification of a Schreibersite precipitate: $\mathrm{P}=24.4 \pm 5.3, \mathrm{Fe}=32.3 \pm 6.6, \mathrm{Co}=$ $1.3 \pm 48.3$, and $\mathrm{Ni}=42 \pm 6.2$ at.\%. This is also within the expected stoichiometry for Schreibersite, $(\mathrm{Fe}, \mathrm{Ni})_{3} \mathrm{P}$. The Cloudy Zone peak is more diffuse than the Kamacite or Plessite peaks with values starting around $15 \%$, a peak around $20 \%$, and then a tail extending towards values of $\sim 30$ at. $\% \mathrm{Ni}$. The peak value agrees with the lower plateau of values seen in the EDX line scan, but excessive smearing is seen between this peak and the Plessite peak. This makes sense since the Cloudy Zone occurs directly between the Plessite phase and the Tetrataenite phase. Therefore, overlap and averaging of these values due to the lateral dimension of both the indentations and the EDX data averaging, as discussed previously, is not too surprising. This averaging is even more pronounced in the Tetrataenite peak, which is very broad in composition space and extends from $\sim 23$ to 42 at.\% nickel. This is quite a bit below the expected 50\% nickel concentration for the FeNi" stoichiometry. While this is an expected result for averaging of a phase with small lateral dimensions, such that there is a lot of probability that indentations occur near/on interfaces, the effect of this averaging is much more pronounced for the Tetrataenite phase than for the Cloudy Zone. Further work is still needed to establish this correlative technique and improve its lateral resolution.

\section{Conclusion}

In this work, mechanical phase mapping was performed using high-speed nanoindentation on the Taza meteorite to determine the properties of the individual phases. Five microstructural phases were characterized in this fragment of the Taza meteorite: Kamacite, Plessite, Tetrataenite, Cloudy Zone, and Schreibersite. This is the first measurement of the mechanical properties of the Tetrataenite $\left(\mathrm{FeNi}^{\prime \prime}\right)$ and the Cloudy Zone (FeNi" + Kamacite nanocomposite) phases. EDX line scans verified the mechanical trends. Several of these phases have very similar hardness or modulus values, but by plotting $H / E$ ratio maps, all phases could be clearly distinguished in the maps. The reason for this superior differentiation in the $H / E$ maps was elucidated using a $2 \mathrm{D}$ histogram of the indentation results or a mechanical phase map. These indentation property maps or mechanical phase maps using 2D histograms are an elegant way to statistically characterize large phases and identify trends in their relationships.

The first direct, point-to-point correlation of EDX and large-scale indentation maps was achieved, but significant further refinement is still possible. This showed excellent agreement for all phases with lateral dimensions much greater than the indentation width. However, EDX results come from an averaged sampled area within/around an indent, so fine-scale phases showed lower than expected stoichiometric compositions due to averaging. More accurate and higher resolution correlative data could be achieved for a depthcontrolled indentation mapping technique.

\section{Materials and Methods}

\section{Materials}

The Taza meteorite [32] (officially designated as NWA 859) is a nickel-iron meteorite classified as a Plessitic Octahedrite, Ungrouped. This means that it contains Widmanstätten bands within a Plessite matrix, but that its trace element composition (Table 3 ) is not consistent with any of the established chemical groupings ( $~ 90 \%$ of nickel-iron meteorites) that have been determined to originate from around a dozen different asteroids. It was discovered in Morocco in 2001 with a main mass of $75.3 \mathrm{~kg}$ and hundreds of smaller fragments. Many of these fragments are oriented in shape from atmospheric ablation, where one surface ablated away and molten surface material flowed and formed flow lines and melted lips as seen in the fragment used in the present work shown in Fig. 7.

The composition of the meteorite is primarily iron, but it contains a high average nickel concentration of $16 \%$ [32]. The microstructure is reported to consist of incompletely

TABLE 3: Trace element concentrations reported [32] for the Taza meteorite.

\begin{tabular}{ll}
\hline \hline Element & Concentration \\
\hline Cobalt & $13.1 \mathrm{mg} / \mathrm{g}$ \\
Nickel & $159.3 \mathrm{mg} / \mathrm{g}$ \\
Copper & $296 \mu \mathrm{g} / \mathrm{g}$ \\
Gallium & $87.0 \mu \mathrm{g} / \mathrm{g}$ \\
Germanium & $2200 \mu \mathrm{g} / \mathrm{g}$ \\
Arsenic & $54 \mathrm{pg} / \mathrm{g}$ \\
Tungsten & $6.8 \mu \mathrm{g} / \mathrm{g}$ \\
Iridium & $2.45 \mathrm{pg} / \mathrm{g}$ \\
Platinum & $37.7 \mu \mathrm{g} / \mathrm{g}$ \\
Gold & $6.52 \mu \mathrm{g} / \mathrm{g}$ \\
\hline \hline
\end{tabular}




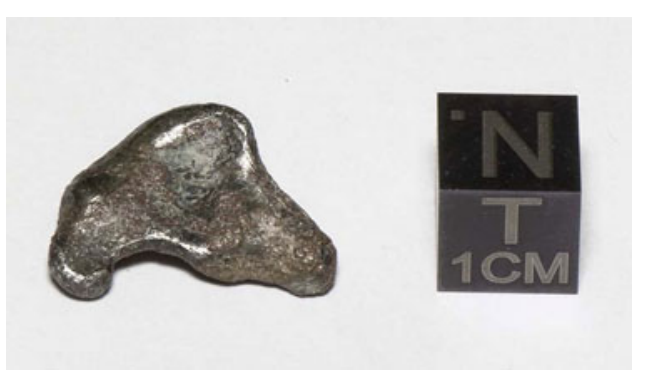

Figure 7: Photograph with a scale marker of the fragment of the Taza meteorite used in the present work.

dissolved Kamacite bands in a Plessite matrix with some hard Schreibersite, $(\mathrm{Fe}, \mathrm{Ni})_{3} \mathrm{P}$, precipitates. This indicates that there is a notable concentration of phosphorus, which is not reported in Table 3. Plessite is a two-phase microstructure, like Pearlite, consisting of undercooled Kamacite precipitating from the high nickel Taenite phase [6].

A small piece of the Taza meteorite was acquired (The Meteorite Exchange, Inc. Tehachapi, CA, USA) and sectioned using an abrasive cutoff saw for this investigation. The sample surface was prepared for nanoindentation by conventional metallographic techniques and polished using successively finer diamond abrasives, finishing with a $60 \mathrm{~nm} \mathrm{SiO}{ }^{2}$ particle suspension. However, the ultimate surface finish was less perfect than desired due to shedding of particles from the frustrum at the edges of the sample into the polishing cloth, contaminating the polishing cloth and resulting in some small surface scratches in the softer phases.

\section{Nanoindentation}

Nanoindentation testing was performed using an iNano nanoindenter (KLA Instruments, Oak Ridge, TN, USA) with a diamond Berkovich indenter. A high-speed nanoindentation technique, NanoBlitz, which allows mapping of a large area using rapid indentation testing, where each test takes $\sim 1 \mathrm{~s}$ and is evaluated by the Oliver and Pharr method [33, 34], was employed to characterize the mechanical properties of the meteorite's microstructure. Preliminary maps of a few hundred indentations were initially performed to identify phases of interest, and grids of 1000 indentations were performed with a progressively decreased load, where each indent was performed at 0.995 of the previous load, to identify general load-depth relationships for the microstructure and the target loads for appropriate grid spacing. Two large indentation maps $(\sim 100$, 000 indents in total) were conducted at an applied load of $15 \mathrm{mN}$ in different areas of the Taza meteorite to characterize regions of interest. A spacing of $5 \mu \mathrm{m}$ was used between indentations to ensure that the suggested indentation depth/spacing ratio of 10 was maintained [35] to avoid excessive interaction between indents. In the case of multi-phase samples, this depth/spacing ratio should be determined for the softest phase in the material, but this does mean that indentations in harder phases penetrate to lower depths. In this case, the chosen load achieved a target depth of $500 \mathrm{~nm}$ to reach a depth in the softer phases where the influence of surface finish was minimized and to ensure that results measured were more representative of bulk behavior.

The iNano system also offers the conventional CSM technique [34], which allows the determination of the hardness, $H$, and elastic modulus, $E$, from the stiffness measured via a small oscillation applied during loading. After identification of the locations of the various phases, a minimum of five CSM indentation tests were performed in each phase to identify the trends in hardness and modulus with depth. These indentations were performed to a load of $50 \mathrm{mN}$ (the maximum load of the iNano) to characterize the phase behavior over the largest range possible.

One important consideration to remember during the analysis of such large volumes of indentation data from multiple phases is the difference between the reduced contact modulus and the Young's modulus. Instrumented indentation allows the measurement of the elastic modulus of a material using the following equations:

$$
\begin{gathered}
E=\left(1-v^{2}\right)\left(\frac{1}{E_{\mathrm{c}}^{*}}+\frac{1-v_{i}^{2}}{E_{\mathrm{i}}}\right)^{-1} . \\
E_{\mathrm{c}}^{*}=\frac{S}{2 \beta} \sqrt{\frac{\pi}{A_{\mathrm{c}}} .}
\end{gathered}
$$

where $E$ is the Young's modulus of the material, $P$ is the load applied, $E_{\mathrm{c}}^{*}$ is the reduced contact modulus, $v$ and $v_{\mathrm{i}}$ are the Poisson's ratio of the sample and the indenter tip, respectively, $E_{i}$ is the Young's modulus of the indenter tip, $\beta$ is a constant to account for an indenter tip without axial symmetry $[7,8]$, and $A_{\mathrm{c}}$ is the projected contact area. This means to correctly determine the Young's modulus of a phase, the Poisson's ratio of that phase needs to be known beforehand or corrected after the fact once the phases have been deconvoluted. For the testing performed here, a Poisson's ratio of 0.3 was assumed for all phases, so the measured modulus values are simply referred to as elastic moduli rather than Young's moduli.

\section{Electron microscopy}

The microstructure of Plessitic meteorites is fairly well understood [6], so phase identification via composition is possible without the necessity for diffraction techniques to further validate the established crystal structures. Scanning electron microscopy was used to observe the nanoindentation maps 
and identify the different phases using two techniques: BSE microscopy (Quanta 200 FEG, Thermo Fisher) and EDX spectroscopy (Octane Elite Super detector, EDAX, Inc., Mahwah, NJ, USA) operated at a $20 \mathrm{kV}$ acceleration voltage with a $4 \mathrm{~ms}$ dwell.

\section{Acknowledgments}

The author thanks K. Kunze (ScopeM, ETH Zurich) for his help in EDX characterization.

\section{References}

1. M. Uehara, J. Gattacceca, H. Leroux, D. Jacob, and C.J. Van Der Beek: Magnetic microstructures of metal grains in equilibrated ordinary chondrites and implications for paleomagnetism of meteorites. Earth Planet. Sci. Lett. 306(3-4), 241 (2011).

2. C.I.O. Nichols, R. Krakow, J. Herrero-Albillos, F. Kronast, G. Northwood-Smith, and R.J. Harrison: Microstructural and paleomagnetic insight into the cooling history of the IAB parent body. Geochim. Cosmochim. Acta 229(1) (2018).

3. H.J. Axon: The metallurgy of meteorites. Prog. Mater. Sci. 13, 183 (1968).

4. J. Yang, J.I. Goldstein, and E.R.D. Scott: Main-group pallasites: Thermal history, relationship to IIIAB irons, and origin. Geochim. Cosmochim. Acta 74(15), 4471 (2010).

5. L.H. Lewis, A. Mubarok, E. Poirier, N. Bordeaux, P. Manchanda, A. Kashyap, R. Skomski, J. Goldstein, F.E. Pinkerton, R.K. Mishra, R.C. Kubic, Jr., and K. Barmak: Inspired by nature: investigating tetrataenite for permanent magnet applications. J. Phys. Condens. Matter 26(6), 064213 (2014).

6. L. Lin, J. Goldstein, and D.B. Williams: Analytical electron microscopy study of the plessite structure in the Carlton iron meteorite. Geochim. Cosmochim. Acta 41(12), 1861 (1977).

7. M. Feller-Kniepmeier and H. Uhlig: Nickel analyses of metallic meteorites by the electron-probe microanalyser. Geochim. Cosmochim. Acta 21(3-4), 257 (1961).

8. F. Dalton: Microhardness testing of iron meteorites (concluded). J. R. Astron. Soc. Can. 45, 162 (1951).

9. F. Dalton: Microhardness testing of Iron meteorites (with plate XI). J. R. Astron. Soc. Can. 44, 185 (1950).

10. A.V. Jain, R.B. Gordon, and M.E. Lipschutz: Hardness of kamacite and shock histories of 119 meteorites. J. Geophys. Res. 77 (35), 6940 (1972).

11. K. Russell, E. Kenik, and M. Miller: Characterization of the Tishomingo meteorite. Surf. Sci. 246(1-3), 292 (1991).

12. E.D. Hintsala, U. Hangen, and D.D. Stauffer: High-throughput nanoindentation for statistical and spatial property determination. JOM 70(4), 494 (2018).
13. J. Roa, P.S. Phani, W.C. Oliver, and L. Llanes: Mapping of mechanical properties at microstructural length scale in WC-Co cemented carbides: Assessment of hardness and elastic modulus by means of high speed massive nanoindentation and statistical analysis. Int. J. Refract. Met. Hard Mater. 75, 211 (2018).

14. G. Guillonneau, M. Mieszala, J. Wehrs, J. Schwiedrzik, S. Grop, D. Frey, L. Philippe, J.-M. Breguet, J. Michler, and

J.M. Wheeler: Nanomechanical testing at high strain rates: New instrumentation for nanoindentation and microcompression. Mater. Des. 148, 39 (2018).

15. F.J. Ulm, M. Vandamme, C. Bobko, J. Alberto Ortega, K. Tai, and C. Ortiz: Statistical indentation techniques for hydrated nanocomposites: Concrete, bone, and shale. J. Am. Ceram. Soc. 90 (9), 2677 (2007).

16. B. Hilloulin, M. Robira, and A. Loukili: Coupling statistical indentation and microscopy to evaluate micromechanical properties of materials: Application to viscoelastic behavior of irradiated mortars. Cem. Concr. Compos. 94, 153 (2018).

17. G. Konstantopoulos, E.P. Koumoulos, and C.A. Charitidis: Testing novel portland cement formulations with carbon nanotubes and intrinsic properties revelation: Nanoindentation analysis with machine learning on microstructure identification.

Nanomaterials 10(4), 645 (2020).

18. M. De Nicolás, H. Besharatloo, J.M. Wheeler, M. de Dios, P. Alvaredo, J. Roa, B. Ferrari, L. Llanes, and E. Gordo: Influence of the processing route on the properties of $\mathrm{Ti}(\mathrm{C}, \mathrm{N})$ Fe15Ni cermets. Int. J. Refract. Met. Hard Mater. 87, 105046 (2020).

19. H. Besharatloo, S. Gordon, T. Rodriguez-Suarez, A. Can,

W. Oliver, L. Llanes, and J. Roa: Small-scale mechanical properties of constitutive phases within a polycrystalline cubic boron nitride composite. J. Eur. Ceram. Soc. 39(16), 5181 (2019).

20. K. Wieczerzak, J. Michler, J.M. Wheeler, S. Lech, R. Chulist,

J. Czub, A. Hoser, N. Schell, and P. Bała: An in situ and ex situ study of $\chi$ phase formation in a hypoeutectic Fe-based hardfacing alloy. Mater. Des. 188, 108438 (2020).

21. B. Vignesh, W. Oliver, G.S. Kumar, and P.S. Phani: Critical assessment of high speed nanoindentation mapping technique and data deconvolution on thermal barrier coatings. Mater. Des. 181, 108084 (2019).

22. M. Sebastiani, R. Moscatelli, F. Ridi, P. Baglioni, and F. Carassiti: High-resolution high-speed nanoindentation mapping of cement pastes: Unravelling the effect of microstructure on the mechanical properties of hydrated phases. Mater. Des. 97, 372 (2016).

23. J. Němeček and J. Lukeš: High-speed mechanical mapping of blended cement pastes and its comparison with standard modes of nanoindentation. Mater. Today Commun. 23, 100806 (2020). 
24. Y. Chen, E. Hintsala, N. Li, B.R. Becker, J.Y. Cheng,

B. Nowakowski, J. Weaver, D. Stauffer, and N.A. Mara:

High-throughput nanomechanical screening of phase-specific and temperature-dependent hardness in AlxFeCrNiMn high-entropy alloys. JOM 71(10), 3368 (2019).

25. Y. Xiao, H. Besharatloo, B. Gan, X. Maeder, R. Spolenak, and J.M. Wheeler: Combinatorial investigation of $\mathrm{Al}-\mathrm{Cu}$ intermetallics using small-scale mechanical testing. J. Alloys Compd. 822, 153536 (2020).

26. J.A. Hartigan and M.A. Wong: Algorithm AS 136: A K-means clustering algorithm. J. R. Stat. Soc. Ser. CAppl. Stat. 28(1), 100 (1979).

27. C. Tromas, M. Arnoux, and X. Milhet: Hardness cartography to increase the nanoindentation resolution in heterogeneous materials: Application to a Ni-based single-crystal superalloy. Scr. Mater. 66(2), 77 (2012).

28. J.-C. Zhao: A combinatorial approach for efficient mapping of phase diagrams and properties. J. Mater. Res. 16(6), 1565 (2001).

29. J.-C. Zhao: A combinatorial approach for structural materials. Adv. Eng. Mater. 3(3), 143 (2001).
30. T. Keil, E. Bruder, and K. Durst: Exploring the compositional parameter space of high-entropy alloys using a diffusion couple approach. Mater. Des. 176, 107816 (2019).

31. R. Skála and I. Císařová: Crystal structure of meteoritic Schreibersites: Determination of absolute structure. Phys. Chem. Miner. 31(10), 721 (2005).

32. S.S. Russell, J. Zipfel, J.N. Grossman, and M.M. Grady: The meteoritical bulletin, no. 86. Meteorit. Planet. Sci. 37, A157 (2002).

33. W.C. Oliver and G.M. Pharr: An improved technique for determining hardness and elastic modulus using load and displacement sensing indentation experiments. J. Mater. Res. 7(6), 1564 (1992).

34. W.C. Oliver and G.M. Pharr: Measurement of hardness and elastic modulus by instrumented indentation: Advances in understanding and refinements to methodology. J. Mater. Res. 19 (1), 3 (2011).

35. P. Sudharshan Phani and W.C. Oliver: A critical assessment of the effect of indentation spacing on the measurement of hardness and modulus using instrumented indentation testing. Mater. Des. 164, 107563 (2019). 